Rochester Institute of Technology

RIT Scholar Works

Presentations and other scholarship

Faculty \& Staff Scholarship

2-25-2019

\title{
Glint Correction of Unmanned Aerial System Imagery
}

Ryan T. Ford

Rochester Institute of Technology

Anthony Vodacek

Rochester Institute of Technology

Follow this and additional works at: https://scholarworks.rit.edu/other

\section{Recommended Citation}

R. T. Ford and A. Vodacek, "Glint Correction of Unmanned Aerial System Imagery," 2019 IEEE Systems and Technologies for Remote Sensing Applications Through Unmanned Aerial Systems (STRATUS), Rochester, NY, USA, 2019, pp. 1-4, doi: 10.1109/STRATUS.2019.8713171.

This Conference Proceeding is brought to you for free and open access by the Faculty \& Staff Scholarship at RIT Scholar Works. It has been accepted for inclusion in Presentations and other scholarship by an authorized administrator of RIT Scholar Works. For more information, please contact ritscholarworks@rit.edu. 


\section{Glint Correction of Unmanned Aerial System Imagery}

\author{
Ryan T. Ford \\ Center for Imaging Science \\ Rochester Institute of Technology \\ Rochester, NY, United States \\ rmf3259@rit.edu
}

\author{
Dr. Anthony Vodacek \\ Center for Imaging Science \\ Rochester Institute of Technology \\ Rochester, NY, United States \\ vodacek@cis.rit.edu
}

\begin{abstract}
Glint in aquatic imagery captured by Unmanned Aerial Systems (UAS) is a limiting factor when performing spectral analysis. It cannot be corrected by methods developed for space-based imaging systems, meaning new approaches are required. Two processes using in-situ radiometric data were developed augmenting an established method for removing atmospheric effects from imagery, the Empirical Line Method (ELM), to remove glint from multispectral UAS imagery. The results of this correction showed good agreement with in-situ spectroradiometer measurements and similar accuracy to atmospherically compensated satellite measurements. The RootMean-Square Error of the UAS retrieved remote sensing reflectance was as low as $0.0004 \mathrm{sr}^{-1}$ and outperformed the traditional ELM.
\end{abstract}

Keywords-Glint Correction, Unmanned Aerial Systems, Remote Sensing, Atmospheric Compensation

\section{INTRODUCTION}

In remote sensing specular reflection from the sun and sky off the water surface, or glint, masks signals relating to waterbodies. Low solar elevation angles as well as high winds and wavy water surfaces during collection lead to high levels of glint within imagery. Accurate spectral analysis for determining waterbody properties such as physics-based inversions or analysis of constituent spectral features can be impossible due to glint. Orienting sensors on unmanned aerial systems (UAS) at a $135^{\circ}$ azimuthal angle from the sun and a $40^{\circ}$ angle from the horizon will minimize glint [1], however post-collection correction is necessary if this orientation cannot be maintained or if imagery is collected in intense glint conditions.

Established methods of glint correction are generally based on statistics or assumptions about thenear-infrared (NIR). Statistical glint correction methods are based on measured wave slope statistics and require a spatial resolution of at least 100 meters to appropriately capture statistically significant wave slope distributions [2]. This makes statistical approaches inappropriate for high spatial resolution UAS imagery. NIR methods assume reflectance in the NIR is solely attributable to glint due to the high absorption of water [3]. This assumption is inaccurate for highly turbid waterbodies due to the increased backscattering of sediments and phytoplankton. New methods without these assumptions are needed to accurately correct the effects of glint in UAS imagery.
In this work two different glint corrections are developed based on the Empirical Line Method (ELM), a method for removing effects of light-atmosphere interactions from imagery. The goals of this work are to evaluate if the developed glint correction methods can accurately remove glint from UAS imagery and to determine if the spectra are recovered with similar levels of accuracy as satellite measurements compensated for atmospheric effects.

\section{METHODS}

\section{A. Study Site and Radiometric Measurements}

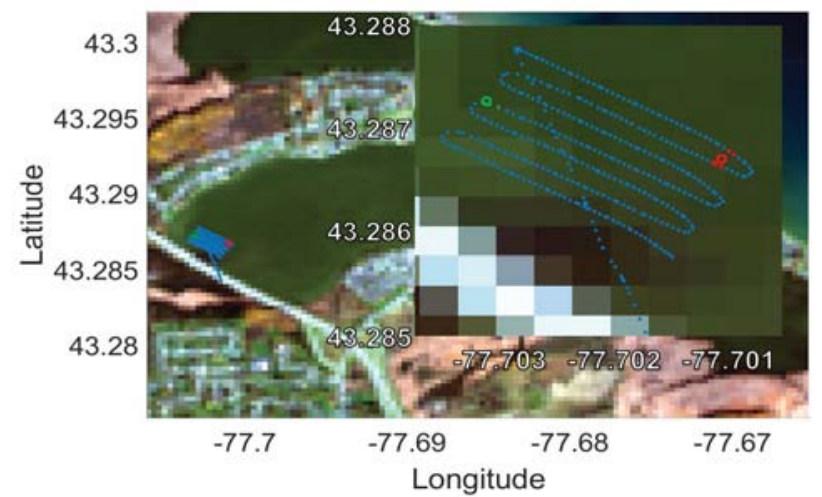

Fig. 1 Landsat 8 image captured over the study area on November 4, 2015. Overlaid are blue dots indicating UAS imagery capture locations (shown larger in the inset). The red dots and circle indicate the image and field measurement locations of LPE, while green indicates LPW.

Paired UAS and satellite imagery was collected in 2015 over Long Pond located in the Rochester Embayment New York, United States (Lat/Long: 43.29 N, 77.69 W). The UAS imagery was collected on November 3 at 18:00 UTC at an altitude of 60 meters. The satellite overpass occurred within 1 day of the UAS collection, taking place November 4 at 16:00 UTC. UAS imagery was only collected over the south end of Long Pond due to flight time limitations. The collection area is shown in Fig. 1. All collections occurred under cloudless sunny skies. Glint was a significant factor of the UAS imagery as wind created a wavy water surface during collection.

Two radiometric measurements were collected within an hour of the satellite overpass. These measurements were performed using an SVC spectroradiometer. These points are 
referred to as Long Pond East (LPE) and Long Pond West (LPW) and indicated in Fig. 1 by red and green respectively. These points were collected in turbid optically deep water and distant enough from shore to avoid any bottom signal. Both measurements were corrected for sun and sky glint. The LPW spectrum was corrected by the method of Mobley (1999) [1]. In this method the sky glint contribution to the water spectrum is determined by multiplying measured sky radiance by a surface reflectance factor, estimated to be $\sim 0.028$ due to the clear sky and low winds during measurement. The determined glint contribution is subtracted from the water measurement, and then normalized by the ratio of radiance to reflectance of a known reference to calibrate to physical values. The Mobley method could not be applied to LPE due to interference in the reference measurement by adjacent objects. LPE was instead corrected using the method from Kutser et al. (2013) [4]. This method fits a power function to the $350-380 \mathrm{~nm}$ and $890-900$ $\mathrm{nm}$ range of the water measurement. The determined power function is assumed to be the glint contribution and subtracted from the measured spectrum to correct for glint.

The output spectra of both methods were in Remote Sensing Reflectance (Rrs, units: $\mathrm{sr}^{-1}$ - inverse steradians), which is defined as the water leaving radiance divided by the downwelled irradiance. Rrs is favorable to water leaving radiance, which is directly related to the pixel values, as it is more invariant to differences in sun/skylight illumination and allows for more consistent spectral analysis. Eight compensated UAS images (five from LPE and three from LPW) were used in analysis.

\section{B. Imaging Sensors}

Imagery was collected by two sensors on different platforms, the Operational Land Imager (OLI) onboard the Landsat 8 satellite, and a six band Tetracam Micro-MCA Snap onboard the UAS. Only the first 5 bands of the Landsat 8 imagery were used for this study. These bands had centers at $443,482,561,654, \& 864 \mathrm{~nm}$ and bandwidths of $16,60,57$, $37, \& 28 \mathrm{~nm}$ respectively. The band centers of the six Tetracam bands were located at 490, 550, 680, 720, 800, and $900 \mathrm{~nm}$. All Tetracam bands had a bandwidth of $10 \mathrm{~nm}$, excluding the 900 $\mathrm{nm}$ band with a bandwidth of $20 \mathrm{~nm}$. The two systems had overlapping coverage in their blue and green bands.

\section{UAS Atmospheric Compensation and Glint Removal}

In order to accurately assess the waterbody from imagery, either the Rrs or water leaving radiance must be known. The signal measured by the UAS contains these values along with the effects of the atmosphere and glint reflected off the water surface. This work primarily focuses on glint correction, but for accurate spectral analysis atmospheric effects must also be removed despite the low flight altitude. The total at aperture radiance $\left(\mathrm{L}_{t}\right)$ can be described by Eq. 1, simplified from [2].

$$
\mathrm{L}_{t}=\tau \mathrm{L}_{\mathrm{w}}+\mathrm{L}_{\mathrm{u}}+\mathrm{Lg}_{\mathrm{g}}=\tau \mathrm{E}_{\mathrm{d}} \rho_{\mathrm{w}}+\mathrm{L}_{\mathrm{u}}+\mathrm{L}_{\mathrm{g}}
$$

Here $\tau$ represents the transmission of light through the atmosphere, $\mathrm{L}_{\mathrm{w}}$ is the water leaving radiance, $\mathrm{E}_{\mathrm{d}}$ is the downwelled irradiance onto the waterbody, $\rho_{\mathrm{w}}$ is the Rrs of the waterbody, $\mathrm{L}_{\mathrm{u}}$ is the atmospheric upwelled radiance, and $\mathrm{L}_{\mathrm{g}}$ is the radiance due to glint. The following sub sections describe each of the compensation methods which were implemented to remove atmospheric and glint effects.

\section{1) Panel-based ELM (Method 1)}

Method 1 was the panel-based ELM [5] implemented using two calibration panels within the scene. This included both a dark and bright panel with known reflectances of about $4 \%$ and $60 \%$ respectively across the VIS-NIR spectrum. The panel reflectances were converted to Rrs by dividing by $\pi$ sr. The Rrs values and mean Digital Counts (DCs) over each panel from the collection altitude were used to determine a linear regression where the slope and y-intercept calculated for each band were related to $\tau \mathrm{E}_{\mathrm{d}}$ and $\mathrm{L}_{\mathrm{u}}$ respectively. In this regression the Rrs values were the dependent variable and the mean DCs were the independent variable, a rearrangement of Eq. 1. The determined factors were used to convert the UAS imagery to Rrs. These factors remain accurate assuming the atmosphere is homogenous across the collection area.

\section{2) Panel-Based ELM with Glint Estimation (Method 2)}

Method 2 is an extended version of the ELM designed to incorporate surface glint. In this method the ELM is performed as described in method 1 to determine the atmospheric variables. The measured Rrs spectrum of a water target was sampled to the spectral response of the Tetracam. The mean band DCs of the brightest water image near the sampling point were determined with the assumption that this image has the most glint. The band sampled Rrs values, average water DCs, and determined atmospheric values were input into the rearranged Eq. 1 in order to solve for the $\mathrm{L}_{\mathrm{g}}$ correction term. This process was performed separately for LPW and LPE imagery due to the non-uniformity of the glint across the collection area.

\section{3) Scene Based ELM (Method 3)}

Method 3 was an implementation of the ELM but with a sampling point in the waterbody used in place of the dark panel. The field measured Rrs spectrum was sampled to the spectral response of the Tetracam bands and the mean band DCs of the brightest water image for each point were calculated. These values were used along with the bright panel DCs and reflectance to perform the ELM. The use of the waterbody and field spectrum allows the effects of glint to be represented in the calculated y-intercept as opposed to using the dark panel in the ELM implemented in method 1.

\section{Satellite Atmospheric Compensation}

The Landsat imagery was compensated for atmospheric effects using three methods designed for inland waterbodies. Multiple compensation methods were implemented to account for the fact that no compensation method retrieves Rrs without error. The first compensation implemented was Atmospheric Correction for OLI Lite (ACOLITE) [6] developed into a software package by the Royal Belgian Institute of Natural Sciences. The second method applied was the Management Unit of the North Sea Mathematical Model (MUMM) compensation [7]. This method was implemented using 
NASA's Ocean Biology Processing Group's SeaDAS imagery processing software. The final compensation method was the Model Based Empirical Line Method (MoBELM) of [8]. This method requires knowledge of the waterbody composition to implement, which was determined using the same methodology as [8]. No glint correction was required for the satellite imagery. Only the satellite pixels that overlapped with the UAS imagery were used to compare the Rrs spectra.

\section{RESULTS AND DISCUSSION}

\section{A. Panel-Based Compensation}

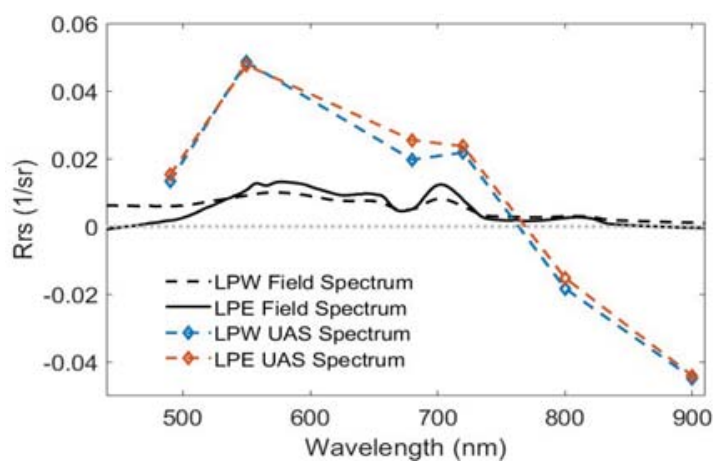

Fig. 2 Comparison of panel-based ELM corrected spectra to field measurements. Note that UAS spectra is multispectral with markers at each band center joined by a dash line for ease of comprehension. The dotted line indicates a Rrs of $0 \mathrm{sr}^{-1}$. The last two bands of both UAS spectra (colored) are both highly overcorrected due to poor ELM coefficient extrapolation. Field spectra (black) are significantly darker than UAS spectra due to glint. LPW UAS spectrum (red) is lower than the LPE spectrum (blue) in the $680 \mathrm{~nm}$ band due to the non-uniformity of the glint.

The normal ELM compensation (method 1) was implemented on the UAS imagery to determine the accuracy of atmospheric compensation without accounting for glint. The coefficients for the ELM compensation were determined using in-scene calibration panels and were applied to the three LPW and five LPE images. The mean Rrs across all bands in all images for both points are plotted in Fig. 2, along with the field measurements.

The UAS retrieved spectra for both locations show significant error when compared to the field measurements. Note that the UAS spectra for both locations appear similar due to the large range of the y-axis. The root-mean-square error (RMSE) between the UAS and band sampled field measurements are shown in Table 1. The last two bands show an overcorrection caused by the Rrs of the water in the NIR being lower than that of the dark panel. The water DCs were therefore out of the calibrated range and overcorrected due to poor extrapolation of the ELM coefficients. The first four bands show a high bias compared to the field spectra due to glint. The largest error occurs in the $550 \mathrm{~nm}$ band, with the Rrs being greater than the field spectra by nearly a factor of five.

The measured field spectra indicate that both locations have similar Rrs values near $680 \mathrm{~nm}$. This is not represented by the UAS measured spectra, which indicate that the Rrs of LPE was greater than that of LPW. This is due to a non-uniform glint radiance across the collection area. Methods 2 and 3 each use water targets in their correction allowing estimation of glint at each point to account for the non-uniformity. This cannot be done with method 1 as the correction factors are determined purely by the panels. This does not indicate that the ELM is inapplicable to water imagery, but it cannot be a total correction in imagery with glint.

\section{B. Augmented ELM Compensations and Comparison with Satellite Spectra}

TABLE I. UAS BAND RRS RMSE

\begin{tabular}{|l|c|c|c|c|c|c|}
\hline & $\mathbf{4 9 0} \mathbf{~ n m}$ & $\mathbf{5 5 0} \mathbf{~ n m}$ & $\mathbf{6 8 0} \mathbf{~ n m}$ & $\mathbf{7 2 0} \mathbf{~ n m}$ & $\mathbf{8 0 0} \mathbf{~ n m}$ & $\mathbf{9 0 0} \mathbf{~ n m}$ \\
\hline LPE 1 & $13.3 \mathrm{e}-3$ & $36.3 \mathrm{e}-3$ & $19.4 \mathrm{e}-3$ & $16.3 \mathrm{e}-3$ & $17.7 \mathrm{e}-3$ & $43.8 \mathrm{e}-3$ \\
\hline LPW 1 & $7.30 \mathrm{e}-3$ & $39.3 \mathrm{e}-3$ & $14.2 \mathrm{e}-3$ & $16.2 \mathrm{e}-3$ & $21.3 \mathrm{e}-3$ & $46.0 \mathrm{e}-3$ \\
\hline LPE 2 & $1.80 \mathrm{e}-3$ & $2.40 \mathrm{e}-3$ & $0.80 \mathrm{e}-3$ & $1.80 \mathrm{e}-3$ & N/A & N/A \\
\hline LPW 2 & $0.52 \mathrm{e}-3$ & $0.29 \mathrm{e}-3$ & $0.61 \mathrm{e}-3$ & $0.47 \mathrm{e}-3$ & N/A & N/A \\
\hline LPE 3 & $1.50 \mathrm{e}-3$ & $1.20 \mathrm{e}-3$ & $1.00 \mathrm{e}-3$ & $1.40 \mathrm{e}-3$ & $0.80 \mathrm{e}-3$ & $1.10 \mathrm{e}-3$ \\
\hline LPW 3 & $0.54 \mathrm{e}-3$ & $0.37 \mathrm{e}-3$ & $0.67 \mathrm{e}-3$ & $0.52 \mathrm{e}-3$ & $0.50 \mathrm{e}-3$ & $0.50 \mathrm{e}-3$ \\
\hline
\end{tabular}

Unlike method 1, methods 2 and 3 both consider the effects of glint. Their retrieved spectra are both shown in Fig. 3. The mean UAS Rrs for each point are shown separately. Table 1 contains the band RMSE values for both methods. As the atmospheric values determined from method 1 are required to implement method 2, the two overcorrected NIR bands were dropped from analysis. Also plotted are the mean satellite spectra calculated from pixels that overlap with the UAS imagery. The RMSE of the satellite Rrs means are shown in Table 2. The field measured spectra are shown in Fig. 3 at full resolution for comparison to both systems.

TABLE II. SATELLITE RRS RMSE

\begin{tabular}{|l|c|c|c|c|c|c|}
\cline { 2 - 7 } \multicolumn{1}{c|}{} & \multicolumn{2}{c|}{ ACOLITE } & \multicolumn{2}{c|}{ MUMM } & \multicolumn{2}{c|}{ MoBELM } \\
\hline & LPE & LPW & LPE & LPW & LPE & LPW \\
\hline $\mathbf{4 4 3} \mathbf{~ n m}$ & $3.44 \mathrm{e}-3$ & $2.80 \mathrm{e}-3$ & $4.16 \mathrm{e}-3$ & $2.20 \mathrm{e}-3$ & $2.38 \mathrm{e}-3$ & $3.61 \mathrm{e}-3$ \\
\hline $\mathbf{4 8 2} \mathbf{~ n m}$ & $3.53 \mathrm{e}-3$ & $1.20 \mathrm{e}-3$ & $2.80 \mathrm{e}-3$ & $1.76 \mathrm{e}-3$ & $2.31 \mathrm{e}-3$ & $2.16 \mathrm{e}-3$ \\
\hline $\mathbf{5 6 1} \mathbf{~ n m}$ & $0.40 \mathrm{e}-3$ & $1.75 \mathrm{e}-3$ & $1.46 \mathrm{e}-3$ & $0.23 \mathrm{e}-3$ & $0.66 \mathrm{e}-3$ & $0.89 \mathrm{e}-3$ \\
\hline $\mathbf{6 5 4} \mathbf{~ n m}$ & $1.97 \mathrm{e}-3$ & $2.13 \mathrm{e}-3$ & $0.322 \mathrm{e}-3$ & $0.56 \mathrm{e}-3$ & $1.13 \mathrm{e}-3$ & $1.44 \mathrm{e}-3$ \\
\hline $\mathbf{8 6 4} \mathbf{~ n m}$ & $3.40 \mathrm{e}-3$ & $1.48 \mathrm{e}-3$ & $2.69 \mathrm{e}-3$ & $0.88 \mathrm{e}-3$ & $1.14 \mathrm{e}-4$ & $0.38 \mathrm{e}-3$ \\
\hline
\end{tabular}

The LPW spectra corrected by methods 2 and 3 matched almost equally well to the measured field spectrum. As a result of taking glint into account, the values for each band were significantly more accurate than those of the panel-based ELM. There is some deviation from the field spectrum, especially in the $680 \mathrm{~nm}$ band caused by spectral averaging. The $680 \mathrm{~nm}$ band averages over the edge of a chlorophyll absorption maximum. These deviations are also present in the corrected spectra from LPE. The last two bands of the spectrum produced by method 3 are preserved as it does not depend on method 1, the panel-based ELM.

Application of methods 2 and 3 to the LPE imagery did not lead to results as consistent as that of LPW. The RMSE values of LPE are greater than that of LPW for both methods. This is primarily due to variability in the imagery which breaks both methods' implicit assumption of homogenous glint. 
Specifically, it is broken by two LPE images taken from a different sensor orientation and therefore sun-target-sensor geometry than the image used to estimate the amount of glint. Similarly using the brightest image for glint estimation is another factor that can break the homogeneity assumption, leading to overcorrection in less affected images. This limitation indicates that if the image glint were to significantly change throughout collection a new estimation would be required. This need for homogeneity further affects method 3 . In its calculation, the y-intercept is representative of both upwelled and glint radiance despite there being no glint from the bright panel. The only glint information arises from the water target used for the correction. Consequently, the further the deviation from a similar Rrs the greater the error in the retrieved value. This compensation should therefore only be applied to areas that are similar in composition to the water imagery used in correction.
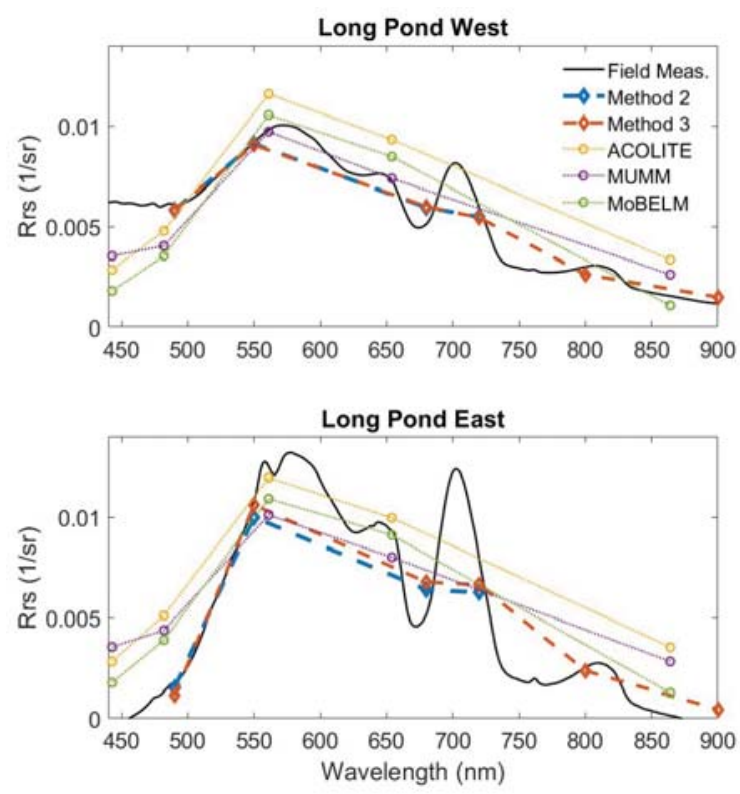

Fig. 3 Results of UAS imagery correction using method 2 (panel-based ELM with glint estimation) and method 3 (scene-based ELM). The top graph shows the results of application to LPW and the bottom LPE with markers at each band center and dashed lines between for ease of comprehension. The dotted lines indicate the 3 different compensated satellite measurements. The solid black lines represent field measurements.

The compensated satellite spectra show general agreement with the measured field spectra. Similar levels of RMSE were determined for each compensation, which were all greater than those of the UAS but comparable. Physical model-based compensations for satellites, such as the MUMM and ACOLITE, often attempt to fit atmospheric conditions based on the imagery and are unable to do so perfectly. This misestimation of atmospheric conditions leads to error in compensation. In the case of the MoBELM, inaccuracy can arise from errors in calculating the bright and dark target spectrum. Assumptions in the methods of correcting the field measurements for glint can also explain the lower accuracy of the satellite spectra. The estimation of the reflectance factor for the Mobley [3] correction may have led to inaccuracy in the LPW measurement. The assumption of no signal while fitting the power function in the Kutser et al. [4] method led to lower Rrs values in the blue and NIR regions where satellite atmospheric compensation is already prone to issues. Despite these factors the UAS and satellite spectra showed similar levels of accuracy, indicating that both methods 2 and 3 are viable for preparing glint affected images for spectral analysis.

\section{CONCLUSION}

This study focused on augmenting the ELM to correct glint in UAS imagery. These augmentations used in-situ data to estimate the contribution of glint to the measured signal. One method focused on using in scene panels to determine the atmospheric effects, and then a glint covered area where the Rrs spectrum was known to determine the effects of glint. The second used a bright target and the known glint area to implement an ELM correction. The first method was less sensitive to changes in Rrs, however was subject to any correction issues caused by the panel-based ELM. The second method was more susceptible to changes in Rrs and glint pattern but were able to retain meaningful information in all bands. Both methods outperformed the traditional ELM, which fails to account for glint. The results for both methods also showed similar levels of accuracy to satellite imagery collected in near time. These methods can improve the ability to build multiplatform datasets and make spectral analysis of glint affected imagery possible.

\section{ACKNOWLEDGMENT}

The authors would like to acknowledge Nina Raqueño, Dr. Carl Salvaggio, Dr. Aaron Gerace, Elizabeth Bondi, and Victoria Scholl for their assistance in collecting this data.

\section{REFERENCES}

[1] C. D. Mobley, "Estimation of the remote-sensing reflectance from above-surface measurements," (in EN), Applied Optics, Vol. 38, Issue 36, pp. 7442-7455, December 1999.

[2] S. Kay, J. Hedley, and S. Lavender, "Sun Glint Correction of High and Low Spatial Resolution Images of Aquatic Scenes: a Review of Methods for Visible and Near-Infrared Wavelengths," (in en), Remote Sensing, Review vol. 1, no. 4, pp. 697-730, October 2009.

[3] J. D. Hedley, A. R. Harborne, and P. J. Mumby, "Technical note: Simple and robust removal of sun glint for mapping shallow-water benthos," (in en), International Journal of Remote Sensing, other vol. 26, no. 10, pp. 2107-2112, February 2007.

[4] T. Kutser, E. Vahtmae, B. Paavel, and T. Kauer, "Removing glint effects from field radiometry data measured in optically complex coastal and inland waters," Remote Sensing of Environment vol. 133, pp. 85-89, June 2013.

[5] G. M. Smith and E. J. Milton, "The use of the empirical line method to calibrate remotely sensed data to reflectance," (in en), International Journal of Remote Sensing, vol. 20, no. 13, Nov 1999.

[6] Q. Vanhellemont and K. Ruddick, "Advantages of high quality SWIR bands for ocean colour processing: Examples from Landsat-8," Remote Sensing of Environment, vol. 161, pp. 89-106, 2015.

[7] K. G. Ruddick, F. Ovidio, and M. Rijkeboer, "Atmospheric correction of SeaWiFS imagery for turbid coastal and inland waters," (in EN), Applied Optics, vol. 39, no. 6, pp. 897-912, 2000-02-20 2000.

[8] J. Concha and J. Schott, "Retrieval of color producing agents in Case 2 waters using Landsat 8," vol. 185, pp. 95-107, November 2016. 